\title{
Impacto da perda dentária na qualidade de vida
}

\author{
Impact of tooth loss in quality of life
}

M aria Elisa de Souza e Silva ${ }^{1}$

Ênio Lacerda Villaça ${ }^{1}$

CláudiaSilami de M agal hães ${ }^{1}$

Efigênia Ferreira eFerreira ${ }^{2}$

${ }^{1}$ Departamento de

Odontologia Restauradora,

FaculdadedeO dontologia,

UniversidadeFederal de

Minas Gerais. Av. Antônio

Carlos 6627/Sala 3339,

Pampulha. 31270-901 Belo

H orizonteM G.

Elisa@ufmg.br

${ }^{2}$ Departamento de

Odontologia Social e

Preventiva, Faculdade de

Odontologia, Universidade

Federal de M inas Gerais.
Abstract In order to evaluatetheimpact of tooth loss in patient's quality of life, 50 volunteers were selected among patients who use the Public $\mathrm{H}$ ealth Services in treatment for their complete denture's placement or replacement. The Oral $\mathrm{H}$ ealth I mpact Profile(OHIP-14) and a socio-demographic data collection wereapplied beforetreatment. The values were founded through the weight of each question in association with Likert's Scale. As higher was the score, higher was the impact in the quality of life. In this survey $82 \%$ were female, $52 \%$ of patients between 41 and 60 years old (average: 59.1), and 34\% married. The higher values of O HIP-14 dimensions in patient's quality of life were: Psychological Discomfort (122), Physical Pain (121), Psychological Disability (113), Physical Disability (109), Functional Limitation (93), Handicap (82) and Social Disability (76). As it could be observed, thelost of teeth or the use of inadequate prosthesis could bring negativeimpacts in life's quality, especially regarding preoccupation, stress with mouth problems and shame, although people perceive minor impacts in social relationships and development of their daily activities. This information can be relevant to prepare dentists to raise their knowledge about edentulous people and how to work with them. Key words Quality of Life, Tooth loss, Impact
Resumo Para avaliar o impacto da perda dentária na qualidade de vida, foram selecionados cinquenta pacientes, usuários do Serviço Público de Saúde, em tratamento para inserção ou substituição do par de dentaduras. Antes do tratamento, aplicou-se o Oral Health Impact Profile (OH IP-14) ea coleta de dados sociodemográficos. O sescores do O HIP-14 foram obtidos por meio do peso de cada pergunta associado à escala de Likert. M aior a pontuação alcançada, maior 0 impacto negativo da dimensão na qualidadedevida. $\mathrm{Na}$ amostra, $82 \%$ eram do gênero feminino, $52 \%$ tinham 41 a 60 anos (média de 59,1 anos), e 34\% eram casados. Para cada dimensão, os valores máximos O H IP-14 foram: desconforto psicológico (122); dor (121); inabilidadepsicológica (113); inabilidadefísica (109); limitação funcional (93); incapacidade (82) e inabilidade social (76). A perda dentária ou o uso de próteses inadequadas implicam impactosnegativosna qualidadedevida, especialmente no que se refere à preocupação, estresse decorrente de problemas na boca eà vergonha. Foi percebido menor impacto no que se refere às relações interpessoais e ao desenvolvimento das atividades rotineiras - dimensão inabilidade social. Estas informações são relevantes para os profissionais, pois ampliam seu conhecimento sobre pessoas desdentadas e melhoram sua capacidade de lidar com elas.

Palavras-chave Qualidade de vida, Perda dental, I mpacto 
Introdução

N o Brasil, o Sistema Ú nico de Saúde (SUS) não tem adequada estrutura nem é suficiente para absorver a demanda por atenção em saúde bucal da população adulta, especialmente nas faixas etária mais avançadas'. Esse é um dos motivos pelos quais dentes que poderiam ser recuperados são extraídos ${ }^{2}$, uma vez que tal alternativa é considerada a mais prática e, também, a mais econômica ${ }^{3}$. A maior parte das pessoas que perdem os dentes vê-se impossibilitada de recompor as perdas por meio de próteses, principalmente devido à falta de recursos financeiros ${ }^{4,5}$. Além disso, os brasileiros que fazem uso de próteses totais removíveis utilizam-nas por um tempo que se estende muito além do prazo recomendado para sua devida substituição ${ }^{1}$.

O levantamento epidemiológico da população brasilei ra divulgado em 1988 expôs, pela primeira vez, a dura realidade da condição de saúde bucal do Brasil. Tal levantamento, que abrangeu apenas a população de até 59 anos, tornou possível verificar 0 alto valor percentual do componente $\mathrm{P}$ (perdido) nos valores do índice CPO-D médio (dentes cariados, perdidos e obturados) dos adultos. $\mathrm{Na}$ população entre 35-44 anos, observou-se o CPO-D de 22,5, e, na faixa que se encontra entre 50-59 anos, 0 de 27,2. 0 componente $P$, nas duas amostras, representou, respectivamente, $66 \%$ e $86 \%$ dos valores relatados ${ }^{6,7}$.

0 inquérito epidemiológico "Levantamento das Condições de Saúde Bucal da população brasileira-SB-Brasil, 2003" mostrou as condições atuais de saúde bucal da população adulta do Brasil. $\mathrm{Na}$ faixa etária de 35-44 anos, o índice CPO-D médio dessa população foi 20,13 , sendo o componente $\mathrm{P}$ (perdido) responsável por um percentual de $66 \%$ do índice. $\mathrm{Na}$ faixa etária de 65-74 anos, o CPO-D foi 27,79 e o componenteP al cançou o percentual de $93 \%$ do índice 7 . Em todo o país, na faixa etária de $30-44$ anos, 30\% das pessoas já são desdentadas e $75 \%$ dos idosos não têm um dente sequer na boca ${ }^{8}$. De maneira contraditória, mais da metade das pessoas com idade superior a 65 anos que participou das entrevistas do levantamento sobre as condições de saúde bucal da população brasileira, quando consultada sobre sua própria saúde bucal, fala e mastigação, classificou-as como boa ou ótima ${ }^{7}$.

Comparando-se os dados revelados, medidos pelo índice CPO-D, em 1988 e em 2003, no quese refereà faixa etária de 35-44 anos, pode-se verificar que houve pouca melhoria da condição de saúde bucal dessa parte da população brasi- leira e que as extrações dentárias são, ainda, largamente utilizadas como tratamento para os problemas bucais $s^{4,9}$.

É possível que alguma alteração desse quadro epidemiológico tenha ocorrido em função da inser ção da odontologia no Programa deSaúde da Família e da criação dos Centros deEspecialidades Odontológicas (CEO), dentro da política do Brasil Sorridente implantada a partir de 2003; no entanto, não existem levantamentos epidemiológicos globais mais atualizados que possam trazer à tona a medida exata desses benefícios. E embora a saúde bucal seja reconhecida como importante, uma considerável parcela da população não tem acesso aos necessários serviços de saúde e a mutilação dentária, resultado da perda dos dentes, acaba por impor às pessoas mudanças físicas, biológicas e emocionais ${ }^{5}$.

$\mathrm{Na}$ rotina diária das pessoas, as alterações produzidas pela perda total dos dentes deveriam se constituir em objeto de preocupação da classe odontológica ${ }^{10}$. No entanto, a abordagem dos profissionais, na maioria das vezes, apenas considera as perspectivas biológicas e restauradoras ${ }^{11}$, ou seja, a recomposição dos dentes deve ser realizada dentro dos melhores princípios da técnica, negligenciando-se as repercussões da perda dental na qualidade de vida dos pacientes ${ }^{4,11}$.

0 presente estudo foi desenvolvido com o objetivo de avaliar o impacto da perda dentária na qualidade de vida de um grupo de pacientes desdentados e identificar as dimensões da qualidade de vida mais afetadas pela condição de saúdebucal.

\section{Métodos}

A Faculdade de O dontologia da Universidade $\mathrm{Fe}$ deral de M inas Gerais (UFM G) é uma das referências para atendimento da população encaminhada pelo Sistema Único deSaúde(SUS) deBelo H orizonte (M G), no que se refere à confecção eà inserção de próteses totais removíveis e absorve, em média, sessenta pacientes por semestre para essetipo de atendimento. 0 tratamento é realizado por meio da disciplina Prótese Total Removível, cujo conteúdo éobrigatório na grade curricular do curso de odontologia dessa faculdade.

Para a composição da amostra deste estudo, deum universo de 66 usuários desdentadosagendados para tratamento na referida disciplina, no primeiro semestre letivo de 2005, foram selecionados cinquenta pacientes, que se enquadraram nos critérios de seleção: já ter perdido todos os 
dentes e estar iniciando o tratamento para a inserção do par de próteses totais removíveis; e no critério de exclusão: o de se caracterizarem como não-leitores. A opção de se trabal har apenas com os pacientes leitores baseou-se no fato de que estes, por serem adultos, compareciam desacompanhados à consulta, não havendo, portanto, como Ihes solicitar as assinaturas de concordância em participar do projeto. Além disso, mesmo que acompanhassem a leitura quando da aplicação do questionário, não seria possível garantir fidedignidade na marcação das suas respostas. Entre os dezesseis pacientes excluídos do estudo, catorze não foram entrevistados, por se enquadrarem no critério de exclusão, eoutros dois não aceitaram participar da pesquisa.

Em se tratando de pesquisa que envolve seres humanos, esta se iniciou após a aprovação do Comitê de Ética em Pesquisa (Parecer $n^{\circ}$ ETIC 442/04)

Os dados do estudo foram coletados com a aplicação do questionário O HIP-14 e através do preenchimento de um formulário que permitiu, inicialmente, a coleta de dados para a caracterização da amostra, incluindo as variáveis gênero, idade, situação laboral, situação civil e tempo de uso de próteses.

No momento seguinte, utilizou-se a forma simplificada do questionário do Oral $\mathrm{H}$ ealth Impact Profile(OHIP-14). 0 questionário OHIP$14^{12,13}$ vem sendo bastante utilizado para indicar os aspectos da qualidade de vida mais afetados pelo estado de saúde bucal eéum forte aliado no estabelecimento de melhores abordagens para atendimento integral ao paciente. Esse instrumento permite indicar as dimensões da qualidade de vida afetadas pela condição de saúde bucal. Os aspectos levantados nas questões aplicadas seguem o modelo conceitual proposto por Locker $^{14}$, que aborda as condições físicas, em que estão incluídos fatores relacionados à locomoção e movimentação, à dor, à alimentação, ao autocuidado e ao descanso; as condições psicológicas, queenglobam o comportamento emocional, o grau de preocupação e a comunicação; e as condições de interação social, em que se observam fatores relacionados ao trabalho, à interação social, à rotina diária e ao entretenimento. Tal abrangência de abordagem é importante e adequada, considerando-se que é perfeitamente possível uma doença produzir impacto em uma ou mais dimensões da vida das pessoas, ou eventualmente, em todas elas ${ }^{14}$.

O formato original do Oral Health Impact Profile(OHIP), composto por 49 questões, já foi validado em várias pesquisas, que confirmaram suas boas propriedades psicométricas e sua eficiência ${ }^{13,15-17}$.

O questionário OHIP-14, denominação conferida à sua forma simplificada, demonstra propriedades similares às do formato original $e$, sendo mais sucinto, requer menos tempo para aplicação, o que favorece sua utilização, inclusive, em serviços de saúde, em processos de avaliação de saúde bucal e qualidade de vida ${ }^{15}$. Também no formato reduzido, esse instrumento foi considerado válido para aplicação em pesquisas relacionadas à qualidade de vida eà saúde bucal da população ${ }^{18}$.

Em 2002, Allen eLocker ${ }^{19}$ desenvolveram uma versão reduzida do OHIP especialmente focada em pessoas desdentadas e verificaram que seu desempenho evalidadeforam compatíveisao instrumento original eatéum pouco melhoresqueo O HIP-14. No entanto, optamos por não utilizar este instrumento tendo em vista que o mesmo não foi testado e validado para a língua portuguesa. Já o OHIP-14 foi testado e validado para uso em função da cultura e da língua locais, medianteuma pesquisa realizada com puérperas, em que se verificou o impacto negativo da dor de dentes na qualidade de vida dessas mulheres ${ }^{20}$. 0 questionário constitui-se de catorze perguntas, duas para cada uma das sete dimensões do instrumento: limitação funcional, dor, desconforto psicológico, inabilidade física, inabilidade psicológica, inabilidadesocial e incapacidade.

Todas as perguntas do questionário OHIP14 procuram relacionar a condição bucal ou das próteses em uso aos temas de cada uma. A dimensão limitação funcional inclui perguntas sobre dificuldade para falar e piora no sabor dos alimentos; na dimensão dor, pergunta-se sobre a sensação de dor e incômodo para comer; na dimensão desconforto psicológico, as perguntas se referem à preocupação e estresse pela condição bucal. 0 prejuízo na alimentação ea necessidade deter de parar desealimentar são os quesitos da dimensão inabilidadefísica, enquanto que na inabilidade psicológica as perguntas referemse à dificuldade para relaxar e ao sentimento de vergonha em função da condição bucal. A dimensão inabilidadesocial inclui perguntas sobre irritação com tercei ros e dificuldade de realizar atividades da rotina diária por causa da condição bucal; e as perguntas que compõem a dimensão incapacidade buscam saber se há a percepção de que a vida tenha piorado e se a pessoa se sentiu totalmente incapaz de desenvolver suas atividades rotineiras. 
Foi feito um pré-teste com aplicação do OHIP-14 a dez pacientes, cujo tratamento iniciou-se em semestre anterior ao da amostra do estudo. N essa oportunidadeverificou-sequenão houve dificuldade no que se refere ao entendimento das questões do instrumento, ao tempo gasto (cerca de vinte minutos) eao local escolhido para o desenvolvimento da atividade.

A coleta dos dados foi realizada nas duas primeiras semanas do tratamento e, para tanto, os pacientes foram acomodados, individualmente, numa sala especialmentereservada para esse fim. A pesquisadora procedeu à leitura de cada pergunta juntamente com o participante. A pós essa leitura, o voluntário escolheu livremente a resposta a cada questão, entre cinco opções, conforme a Escala de Likert ${ }^{21}$ - sempre, com frequência, às vezes, raramente e nunca - e acompanhava a marcação indicada. O s dados foram lançados no Programa Epi Info, versão 2005, que permitiu o cálculo das médias e percentuais para caracterização da amostra, de acordo com as variáveis listadas.

Posteriormente, os dados foram exportados com vistas à composição de planilhas no programa Excel da M icrosoft, versão 2001. A utilização desse programa permitiu a ordenação dos dados de acordo com o objetivo a ser verificado, bem como o cálculo do valor OHIP-14, em pontos para cada paciente entrevistado, em cada dimensão, e na somatória delas. As pontuações foram obtidas a partir da aplicação do escalonamento Likert em associação com o peso de cada pergunta, conforme preconizaram os idealizadores do instrumento ${ }^{12}$. A cada categoria, foi atribuído um valor entre 1 e 5 , que era multiplicado pelo peso da questão. A resposta sempre recebeu o maior número de pontos, 5; com frequência, 4 pontos; às vezes, 3 pontos; raramente, 2 pontos e nunca recebeu 1 ponto.
No questionário OHIP-14, uma vez que cada duas perguntas correspondem a uma dimensão, chegou-se à pontuação de cada dimensão por meio da soma dos valores OH I P-14 das duas perguntas correspondentes. Na hipótese de uma dimensão atingir o máximo possível de pontuação OHIP-14, sua somatória chegaria a 250 pontos.

\section{Resultados}

A amostra foi composta de $82 \%$ do gênero feminino e $18 \%$ do gênero masculino. Em um intervalo de 37-83 anos, a média de idade dos participantes foi 59,1 anos (+ 11,9 anos). As características sociodemográficas da amostra podem ser visualizadas na Tabela 1 . Apenas $24 \%$ da amostra (12) estavam em atividade laboral; os demais eram aposentados ou não trabalhava.

O Gráfico 1 mostra os valores obtidos para cada dimensão, após a apuração das respostas.

Observa-se que desconforto psicológico, dor e inabilidade psicológica foram as dimensões de maior impacto na qualidade de vida das pessoas entrevistadas. No entanto, tomando-se a pontuação al cançada como referência, a perda dentária total não representou um forte obstáculo à interação social dessas mesmas pessoas.

Tanto para os dez pacientes que alcançaram os maiores valores do OHIP-14 quanto para os dez pacientes que atingiram os menores valores, as perguntas que obtiveram as maiores pontuações foram as referentes a sentir-se incomodado ao comer algum alimento por causa de problemas com sua boca ou com sua dentadura - dimensão dor - e o sentimento de vergonha por causa de sua boca ou de sua dentadura - dimensão inabilidade psicológica.

A maior partedos entrevistados relatou nunca ter enfrentado problema com relação às dimen-

Tabela 1. Caracterização da amostra que respondeu ao Questionário Oral Health Profile -OHIP-14, quanto à faixa etária, situação civil, uso de prótese e tempo de uso de prótese. Belo Horizonte (M G), 2005.

\begin{tabular}{|c|c|c|c|c|c|c|c|}
\hline \multirow{2}{*}{$\frac{\text { Gênero }}{\text { Faixa etária }}$} & \multicolumn{3}{|c|}{ Homem } & \multicolumn{3}{|c|}{ Mulher } & \multirow[b]{2}{*}{ Total $(\%)$} \\
\hline & $<40$ anos & 41 a 60 anos & $>61$ anos & $<40$ anos & 41 a 60 anos & $>61$ anos & \\
\hline Distribuição por faixa etária & 0 & 5 & 4 & 1 & 21 & 19 & $50(100)$ \\
\hline Vive com companheiro & 0 & 3 & 3 & 1 & 11 & 4 & $22(44)$ \\
\hline $\mathrm{N}$ ão vive com companheiro & 0 & 2 & 1 & 0 & 10 & 15 & $28(56)$ \\
\hline U sa pelo menos uma PTR & 0 & 4 & 3 & 0 & 13 & 15 & $35(70)$ \\
\hline Usa PTR há mais de 10 anos & 0 & 0 & 2 & 0 & 11 & 15 & $28(80)$ \\
\hline
\end{tabular}




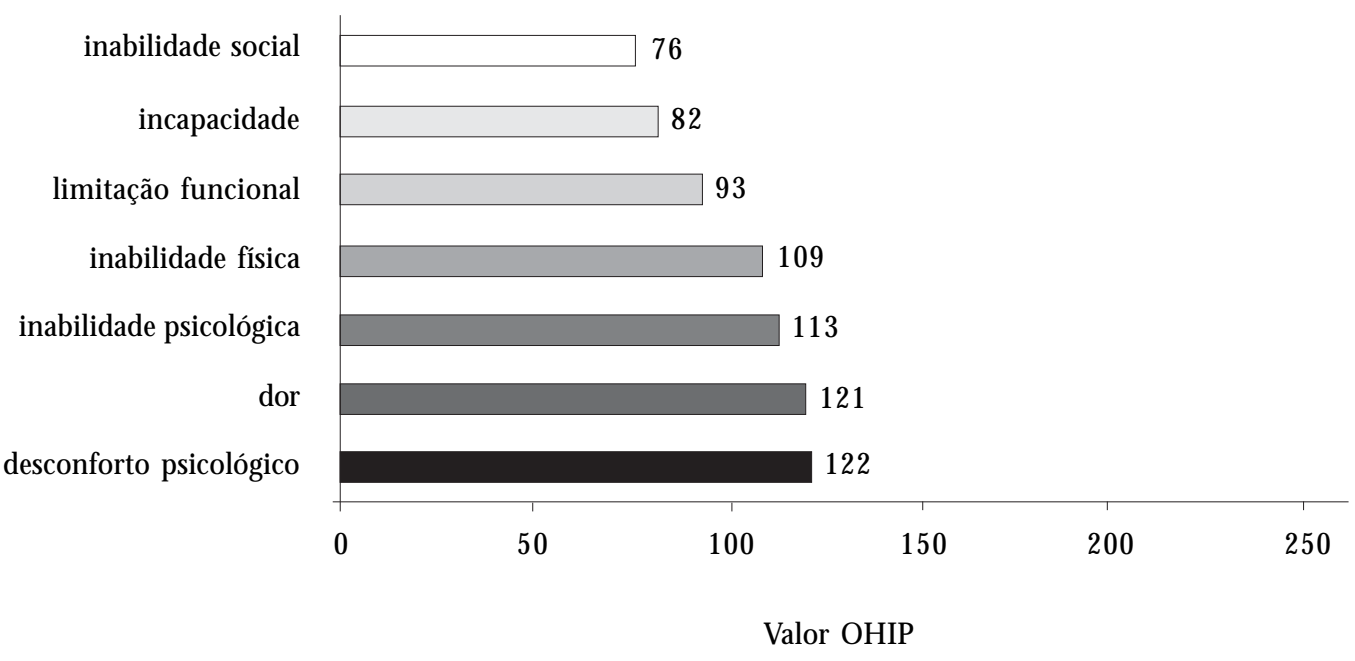

Gráfico 1. Total de pontos por dimensão do questionário Oral Health Profile - OHIP-14. Belo Horizonte (M G), 2005.

sões incapacidade ( $80 \%)$, cujas perguntas referem-se à percepção de que a vida ficou pior por causa de problemas com seus dentes, sua boca ou dentadura e se ficou totalmente incapaz de fazer suas atividades diárias por causa de problemas com seus dentes, sua boca ou dentadura; inabilidade social $(79 \%)$, que inclui questionamentos sobre a pessoa ter ficado irritada com outras pessoas por causa de problemas com seus dentes, sua boca ou dentadura e tido dificuldades de realizar suas atividades diárias por causa de problemas com seus dentes, sua boca ou dentadura, e a dimensão limitação funcional (63\%), cujas questões levantam a percepção sobre problemas para falar alguma palavra por causa de problemas com seus dentes, sua boca ou boca dentadura e se o sabor dos alimentos ficou pior por causa de problemas com seus dentes, sua boca ou dentadura.

Ao se considerar a frequência da resposta sempre (Tabela 2), permanecem como dimensões de maior impacto na qualidade de vida desconforto psicológico (17\%), inabilidade psicológica ( $17 \%)$, inabilidade física ( $10 \%$ ) e dor (9\%).

Entre as catorze questões aplicadas, a que apresentou o maior percentual da resposta sem- prefoi a que se refereà experiência do sentimento de vergonha por causa da boca - dimensão inabilidadepsicológica. Entre os dez pacientes com os maiores valores de OHIP-14, nove escolheram esta opção.

Na Tabela 2, apresentam-se as frequências das respostas de menor e maior valor em cada dimensão considerada. Além da vergonha por causa da boca, entre todos os pacientes entrevistados, a questão sobre incômodo para comer por causa de problemas com a boca ou com a dentadura - dimensão dor -, a questão sobre preocupação com a situação da boca ou com a dentadura - dimensão desconforto psicológico - e a que se refere à impressão de que a alimentação ficou pior por causa de problemas com a boca ou com a dentadura - dimensão inabilidade física-, configuram-se como aquelas cujas respostas foram mais enfáticas, ficando a escol ha sobre as opções sempre e com frequência em expressivo percentual de vezes - 36\%, 38\% e $34 \%$, respectivamente.

$\mathrm{M}$ aior percentual de respostas de baixo impacto foi apurado relativamente à questão que aborda a incapacidade para a realização das tarefas. A este quesito, $98 \%$ da amostra, ou seja, 49 dos 50 entrevistados, responderam nunca. 
Tabela 2. Frequência simples das respostas de menor e maior impacto e moda para as sete dimensões estudadas. Belo Horizonte (MG), 2005.

\begin{tabular}{lcc}
\hline \multicolumn{1}{c}{ Dimensão OHIP-14 } & $\begin{array}{c}\text { Frequência menor } \\
\text { valor atribuído - \% } \\
1 \text { (Nunca) }\end{array}$ & $\begin{array}{c}\text { Frequência maior } \\
\text { valor atribuído - \% } \\
\text { (Sempre) }\end{array}$ \\
\hline Limitação funcional & 63 & 2 \\
Dor & 48 & 9 \\
Desconforto psicológico & 56 & 17 \\
Inabilidade física & 50 & 10 \\
Inabilidade psicológica & 47 & 17 \\
Inabilidade social & 79 & 4 \\
Incapacidade & 80 & 8 \\
\hline
\end{tabular}

\section{Discussão}

A avaliação do impacto da saúde bucal na qualidade de vida das pessoas realizada a partir da utilização de um questionário estruturado como - OHIP-14 apresenta vantagens, como a possibilidade de o pesquisador explicar os objetivos da pesquisa, orientar o preenchimento do instrumento e obter dados mais uniformes e úteis. No entanto, essetipo de instrumento tem limitações como o risco de distorções, por insegurança nas respostas devido à presença do entrevistador e por menor liberdade nas respostas, uma vez que elas já vêm preestabelecida ${ }^{22}$.

Nos últimos anos, vem sendo observada a preocupação em se avaliar o impacto da perda dental e da utilização de próteses removíveis na qualidade de vida das pessoas e os resultados das pesquisas desenvolvidas nessa direção demonstram que as repercussões são relevantes e não podem ser desconsideradas ${ }^{23-26}$. Grande atenção, deve, pois, ser dispensada aos pacientes, especialmente no momento de preparo dos mesmos para a perda total dos dentes, no atendimento das suas expectativas com a inserção das próteses e no seu posterior monitoramento ${ }^{27}$.

A perda dentária pode ser considerada como uma saída para o fracasso de um tratamento conservador anteriormente realizado $0^{28}$ e pode se constituir em um evento de forte impacto, que, além de causar danos funcionais, é capaz de desequilibrar a organização psíquica e social das pessoas ${ }^{11}$.

No presente estudo, foram incluídos como voluntários pacientes desdentados, com necessidade de reposição protética. 0 fato da maioria da amostra ter sido formada por mulheres (82\%) é comum nos levantamentos realizados nas clínicas da Faculdade de O dontologia da UFM G 29,30. Este dado não está relacionado à prevalência de uma situação num determinado grupo, mas à disponibilidade de tempo para frequentar clínicas de ensino, na maioria das vezes, em horários diurnos ecuja expectativa de conclusão do tratamento, em função da inexperiência dosalunos, é de um tempo maior.

A maior parte do grupo devoluntários (98\%) apresentava idade superior a quarenta anos. 0 expressivo percentual de indivíduos (52\%) entre quarenta anos e sessenta anos está relacionado a vários fatores, sobretudo socioeconômicos, dentre eles, a falta de acesso ao tratamento, vivenciada pela grande maioria da população brasileira, motivo pelo qual é frequente, nessa população, a perda dentária precoce ${ }^{2}$. M esmo sendo considerada uma alternativa mais prática e econômica ${ }^{3}$, a reabilitação protética para desdentados, ofertada no rol de procedimentos da atenção secundária, équaseinexistenteno serviço público einsuficiente para atender à demanda da populaçãa ${ }^{1}$.

Defato, até2004, apenas $2,8 \%$ do tratamento realizado no SUS era de maior complexidade. Com a inserção da odontologia no Programa de Saúde da Família e com a implantação dos Centros Especializados O dontológicos (CEO) e dos Laboratórios Regionais de Próteses Dentárias (LRPD), integrantes do Projeto Brasil Sorridente, a partir de 2004, é possível que ocorra, em médio prazo, uma redução da demanda por tratamento mais complexo, principalmente próteses totais, ainda bastante expressiva em nosso país. Ressalte-seque, na atualidade, para boa parte da população com mais de 44 anos, a solução para os problemas de saúde bucal deve ser a inserção de próteses totais removíveis 8 .

Braga et al. ${ }^{1}$ recomendam que as próteses totais removíveis sejam trocadas, no mínimo, a cada cinco anos, em função do desgaste do material utilizado - acrílico - edos malefícios que podem decorrer desse desgaste. No entanto, se o acesso à atenção odontológica para a reabilitação é escasso, pode-se dizer que ele inexiste para a manutenção da prótese. Considerando-se os pacientes da amostra estudada, observou-seque $80 \%$ dos que já usavam prótese estavam utilizando a mesma prótese há mais de dez anos, o queéusual à população brasileira.

Entre todas as perguntas do instrumento aplicado (OHIP-14), a que provocou as respostas mais enfáticas é a que argúi sobre o paciente se sentir envergonhado por causa dos problemas com sua boca ou com sua dentadura - di- 
mensão inabilidade psicológica - , o que corrobora o resultado de outras pesquisas que abordam a dificuldade de aceitação e o sentimento de humilhação que envolve uma pessoa desdentada ${ }^{5,11,23,25}$. Mesmo nos pacientes cujas pontuações O HIP-14 foram menores, o que indica menor percepção do impacto da condição bucal na sua qualidade de vida, a questão da vergonha é bastante ressaltada. A função estética dos dentes é, muitas vezes, considerada mais importanteque a função mastigatória. Sob o ponto de vista subjetivo, já se verificou que a perda de elementos localizados mais posteriormente na boca tem pouco ou nenhum impacto na vida das pessoas, diferentemente da perda dental total ou de dentes anteriores, que motiva as pessoas a buscar tratamento para substituição deles ${ }^{31}$.

A ausência total dos dentes também implicou impacto negativo no que se refere à alimentação - inabilidade física. A estabilidade das próteses, especial menteas mandibulares, euma adequada mastigação dos alimentos podem ser citadas como fatores que muito interferem na qualidade de vida de pacientes desdentados, levando a uma pior condição de saúde geral ${ }^{32,33}$. Embora tais estudos apontem nessa direção, o estudo realizado por Gomes et al. ${ }^{34}$ afirma quenão há uma relação direta entre a ausência de dentes e uma pior condição de saúde geral.

A ausência total dos dentes e a utilização de próteses totais removíveis inadequadas podem causar efeitos como dificuldade para relaxar, embaraço, restrição em comer determinados alimentos e até perda da vontade de sair de casa ${ }^{25,35}$. M esmo que não tenha, ainda, sido comprovada uma forte relação entre a presença de sintomas de disfunção craniomandibular e a ausência ou uso de próteses totais, os profissionais devem estar sempre atentos à possibilidade de se estabelecer um plano correto, que resultena inserção de próteses mais confortáveis e adequadas ${ }^{36-38}$.

Embora a condição de saúde bucal cause impacto em vários aspectos da qualidade de vida das pessoas ${ }^{39}$, para esse grupo, na dimensão inabilidade social, que se refere à interação social e ao desenvolvimento das atividades rotineiras, o prejuízo observado, representado pela menor pontuação alcançada, foi bem menor que para as demais dimensões. N este estudo, comprovou-se que os aspectos que mais incomodam os pacientes desdentados são a vergonha de não ter dentes - dimensão da inabilidadepsicológica-, o incômodo ao comer - dimensão dor - e a preocupação com a boca dimensão desconforto psicológico - e prejuízo na alimentação - dimensão inabilidade física.
É interessante observar, ainda, que a resposta mais frequente para todas as perguntas do OHIP14 foi nunca, o que corrobora os resultados obtidos em trabal hos que apontam para o fato de as pessoas terem precária percepção de seus problemas bucais, como se estes fossem inevitáveis nas faixas etárias mais avançadas ${ }^{40}$. A extração total dos dentes representa, muitas vezes, uma solução definitiva para a questão da dor ${ }^{4,28}$.

M esmo a dimensão que apresentou a maior pontuação OHIP-14, desconforto psicológico, não chega à metade do maior valor possível de se atingir. No entanto, os problemas advindos da falta dos dentes podem abranger não só a limitação da capacidade mastigatória ${ }^{41}$ e capacidade de fonação, mas também o comprometimento da aparência estética ${ }^{11}$ ea descaracterização da identidade facial, retratando um quadro social de menor privilégio, o que leva o paciente a se tornar uma pessoa com dificuldades de aceitação social plena, portador de um estigma ${ }^{42,43}$.

0 fato de algumas dimensões serem maisfrequentes e importantes para o grupo estudado não invalida ou torna as outras menos importantes. Individualmente, é possível que a ausência dos dentes produza maior impacto em uma ou mais dimensões na vida de uma pessoa, ou, eventualmente, em todas elas ${ }^{14}$.

Apenas a abordagem quantitativa é, porém, insuficiente para permitir um bom entendimento dos sentimentos que envolvem as pessoas com perda total dos elementos dentais, ainda que possa, sem dúvida, contribuir para delinear caminhos que possibilitem um aprofundamento no conhecimento ena interpretação desses sentimentos, bem como de suas consequências na qualidade de vida das pessoas. No entanto, a utilização de um instrumento como este, antes do tratamento para a confecção de próteses totais, pode orientar o profissional com relação ao impacto da perda dentária para as pessoas e os cuidados quese deve ter na sua orientação, acolhida eatendimento e proservação.

\section{Conclusão}

Verificou-se, no grupo de pessoas desse estudo, que a ausência de dentes ou a utilização de próteses inadequadas pouco interfere na capacidade delas realizarem suas atividades diárias e de se inter-relacionarem no meio em que vivem, embora provoquem impactos negativos em algumas dimensões da qualidade de vida, queforam desconforto psicológico, dor e inabilidade psicológica. 
Essas informações são importantes para uma adequada capacitação dos profissionais responsáveis pela aten ção aos pacientes que experimentaram ou estão por experimentar a perda total ou parcial, dos dentes. É mister que os aspectos psicológicos e as questões subjetivas que envolvem cada situação sejam considerados tão essenciais quanto o foco técnico.

\section{Colaboradores}

MESSilva idealizou eredigiu o projeto de pesquisa, realizou as entrevistas, o levantamento e análise dos dados, o levantamento bibliográfico e a estruturação, a redação da primeira versão do artigo e da versão final do texto. E Ferreira eFerreira auxiliou na elaboração da metodologia, na revisão bibliográfica, na análise dos resultados e redação da versão final do artigo. CS M agalhães participou da análise dos resultados e da redação da versão final do artigo. EL Vilaça contribuiu para a organização e a análise dos dados, bem como para o processamento no programa Epi Info e para a redação da versão final do texto.

\section{Agradecimentos}

Os autores agradecem ao Professor Ricardo $\mathrm{Hi}$ roshi Takahashi pelo apoio técnico e pela contribuiçãa no momento da realização dos cálculos matemáticos para apuração das pontuações obtidas.

\section{Referências}

1. Braga SRS, Telarori Jr. R, Braga AS, Catirse ABEB. Avaliação das condições e satisfação com as próteses em idosos na região Central do Estado de São Paulo (Brasil). Rev Odontol UNESP 2002; 31(1):39-48.

2. Guimarães $M M, M$ arcus $B$. Expectativa de perda de dente em diferentes classes sociais. REVCROMG 1996; 2(1):16-20.

3. Pinto VG. Saúde bucal coletiva. São Paulo: Santos; 2000.

4. Vargas AMD, Paixão HH. Perda dentária e seu significado na qualidade de via de adultos usuários de serviço público de saúde bucal do Centro de Boa Vista, em Belo H orizonte. Cien Saude Colet 2005; 10(4):1015-1024.

5. Ferreira AAA, Piuvezam G, Werner CWA, Alves MSCF. A dor e a perda dentária: representações sociais do cuidado à saúde bucal. Cien Saude Colet 2006; 11(1):211-218.

6. Pinto VG. Saúde bucal: a odontologia social e preventiva. São Paulo: Santos, 1989.

7. Brasil. Ministério da Saúde. Projeto SB Brasil 2003: condições de saúde bucal da população brasileira 20022003: Resultados Principais. Brasília: Ministério da Saúde; 2004.

8. Pucca Jr. GA. A política nacional de saúde bucal como demanda social. Cien Saude Colet 2006; 11(1):243-246.

9. Colussi CF, Freitas SFT. Aspectos epidemiológicos da saúde bucal do idoso no Brasil. Cad Saude Publica 2002;18(5):1313-20.

10. Chianca TK, Deus MR, Dourado AS, Leão AT, Vianna RBC. El impacto de la salud bucal en calidad de vida. Rev Fola/oral 1999; 16:96-102.

11. Wolf SMR. O significado da perda dos dentes em sujeitos adultos. Rev Assoc Paul Cir Dent 1998; 52(4):307-315.

12. Slade GD, Spencer AJ. Development and evaluation of the oral health impact profile. Community Dent Health 1994; 11(1):3-11.

13. Slade GD, Hoskin GW, Spencer AJ. Trends and fluctuation in the impact of oral conditions among older adults during a one year period. Community Dent Oral Epidemiol 1996: 24(5):317-321. 
14. Locker D. M easuring oral health: a conceptual framework. Community Dent Health 1988; 5(1):5-13.

15. Slade GD. Derivation and validation of a shortform oral health impact profile. Community Dent Oral Epidemiol 1997; 25(4):284-290.

16. Allen PFA. A comparison of the validity of generic and disease-specific measures in the assessment of oral health-related quality of life. Community Dent Oral Epidemiol 1999; 27(5):344-352.

17. Broder HL, Slade G, Caine R, Reisine S. Perceived impact of oral health conditions among minority adolescents. J Public Health Dent 2000; 60(3):189192.

18. Wong MC, Lo EC, MCMillan AS. Validation of a Chinese version of the Oral Health Impact profile (OHIP). Community Dent Oral Epidemiol 2002; 30(6):423-430.

19. Allen F, Locker D. A modified short version of the oral health impact profile for assessing health-related quality of life in edentulous adults. Int J Prosthodont 2000; 15(5):446-450.

20. Oliveira BH, Nadanovsky P. Psychometric properties of the Brazilian version of the Oral $\mathrm{H}$ ealth Impact Profile - short form. Community Dent Oral Epidemiol 2005; 33(4):307-314.

21. Babbie E. M étodos de Pesquisa de Survey. Belo Horizonte: UFM G; 1999.

22. Cummings SR, Stewart AL, Hulley SB. Designing questionnaires and data collection instrument. In: Hulley S, Cummings SR, Browner WS, N ewman TB, $\mathrm{H}$ earst $\mathrm{N}$, editors. Designing clinical research: an epidemiologic approach. $2^{\text {nd }}$ ed. Philadelphia: Lippincott Williams \& Wilkins; 2001. p. 231-245.

23. Fiske J, Davis DM, Frances C, Gelbier S. The emotional effects of tooth loss in edentulous people. $\mathrm{Br}$ Dent J 1998; 184(2):90-93.

24. Klages U, Esch M, Wehrbein H. Oral Health Impact in patients wearing removable prostheses: relation to somatization, pain sensitivity, and body consciousness. Int J Prosthodont 2005; 18(2):106-111.

25. Jones JA, Orner MB, Spiro A, Kressin NR. Tooth loss and dentures: patient's perspectives. Int Dent J 2003; 53(5):327-334.
26. John MT, Koepsell TD, Hujoel P, LeResche L, Micheelis W. Demographic factors, denture status and oral health-related quality of life. Community Dent Oral Epidemiol 2004; 32(2):125-132.

27. Freitas KM, Oliveira PEF, Paranhos HFO, Muglia VA, Pardini LC. Avaliação do grau de satisfação de pacientes reabilitados com próteses totais convencionais. Rev Paul Odontol 2001; 23(6):25-28.

28. M endonça MT. Mutilação dentária: concepções de trabalhadores rurais sobre a responsabilidade pela perda dentária. Cad Saude Publica 2001; 17(6):15451547.

29. Centro de Apoio, Seleção e Encaminhamento do U suário da Faculdade de Odontologia da Universidade Federal de Minas Gerais. Relatório de Controle de Frequência de Pacientes das Clínicas da Faculdade de O dontologia da UFM G. Belo H orizonte: UFM G; 2005.

30. Centro de Apoio, Seleção e Encaminhamento do U suário da Faculdade de Odontologia da Universidade Federal de M inas Gerais. Relatório de Controle de Frequência de Pacientes das Clínicas da Faculdade de O dontologia da UFM G. Belo H orizonte: UFM G; 2005.

31. Elias AC, Sheiham A. The relationship between satisfaction with mouth and number and position of teeth. J Oral Rehabil 1998; 25(9):649-661.

32. Agerberg C, Carlsson GE. Chewing ability in relation to dental and general health. Acta O dontol Scand 1981; 39(4):147-153.

33. Oliveira TRC, Frigerio M LMA. Association between nutrition and the prosthetic condition in edentulous elderly. Gerodontology 2004; 21(4):205-208.

34. Gomes VN, Frigerio M LM A, Fidelix M. Bone mass index analisys in elderly people before and after change prosthesis. Gerodontology 2006; 23(3):187-191.

35. Marcenes W, Steele JG, Sheiham A. A relação entre estado dentário, seleção alimentar, ingestão de nutrientes, estado nutricional e índice de massa corporal em idosos. Cad Saude Publica 2003; 19(3):809-815.

36. Ribeiro RA, Mollo Jr. FA, Pinelli LAP, Arioli Jr. JN, Ricci WA. Prevalência de disfunção craniomandibular em pacientes portadores de próteses totais duplas e pacientes dentados naturais. Cienc Odontol Bras 2002; 5(3):84-89. 
37. Arana ARS, Moura JA, Kignel S. Prevalência de disfunção craniomandibular entre os pacientes portadores de prótese total. Rev Paul Odontol 2000; 22(5):4-6.

38. Ribeiro RA, Mollo Junior FA, Arioli Jr. JN, Pinelli LAP. Sintomas de disfunção craniomandibular. RGO 2003; 51(2):123-126.

39. Biazevic MGH, Michel-Cosato E, lagher F, Pooter CE, Correa SL, Grasel CE. Impact of oral health on quality of life among the elderly population of J oaçaba, Santa Catarina, Brazil. Braz Oral Res 2004; 18(1):85-91.

40. Silva SRC, Fernandes RAC. Autopercepção das condições de saúde bucal por idosos. Rev. Saude Publica 2001; 35(4):349-355.

41. M CGrath C, Bedi R. Can dentures improve the quality of life of those who have experienced considerable tooth loss? J Dent 2001; 29(4):243-246.

42. Goffman E. Estigma: notas sobre a M anipulação da Identidade Deteriorada. 4a ed. Rio de Janeiro: Guanabara-Koogan; 1988.

43. Appollonio I, Carabellesse C, Frattola A, Trabucchi M. Dental status, quality of life, and mortality in an older community population: A multivariate approach. J Am Geriatr Soc 1997; 45(11):1315-1323.

Artigo apresentado em 27/07/2006

Aprovado em 08/01/2007

Versão final apresentada em 04/04/2008 